\title{
Collagen-like sequences in phages and bacteria
}

\author{
JUERGEN ENGEL*1 and HANS PETER BÄCHINGER ${ }^{2}$ \\ 'Department of Biophysical Chemistry, Biozentrum, University of Basel, \\ Klingelbergstr. 70, CH-4056 Basel, Switzerland \\ ${ }^{2}$ Research Unit, Shriners Hospital for Children and the Department of \\ Biochemistry and Molecular Biology, Oregon Health Science University, \\ Portland, Oregon, 97201, USA \\ e-mail: engel@ubaclu.unibas.ch; HPB@shcc.org
}

\begin{abstract}
Sequences with glycine in every third position have been detected in DNA derived sequences of proteins in phages and bacteria and it was suggested that these regions trimerise to collagenous structures. Related sequences are found in proteins of mollusks and slime mold. The sequences contain a much lower fraction of proline than mammalian collagens and it is unknown how many of the prolines, if any, are converted to hydroxyproline. Therefore, for triple helix formation other stabilizing interactions than those known for mammalian collagens are required. Strikingly, aspartate and asparagine are abundant in collagen-like sequences of phage tail fibre proteins and of related sequences in nacrein of oyster pearls suggesting a possible stabilization by calcium binding.
\end{abstract}

Keywords. Triple helix; stabilization; evolution.

\section{Introduction}

In the past, research on collagens was focused on mammalian collagens of which 19 members, collagen I to XIX, are known today ${ }^{1,2}$. This classification does not include collagens which are not directly connected to the extracellular matrix, like C1q and the collectins $^{3}$. All collagens contain by definition collagen domains but also a large variety of other domains. The number of genes is higher than the number of collagens because each collagen consists of three chains, which are often different. For the formation of the collagen triple helix the occurrence of glycine in every third position is a prerequisite, because larger side chains than hydrogen atoms would interfere with the packing of three polyproline helices to the triple helix ${ }^{1,4}$. Therefore collagenous sequences can be easily recognized by their Gly-Xaa-Yaa repeat. Another characteristic feature of most collagenous sequences is the occurrence of hydroxyproline in Y-positions. It was recognized that this residue is essential for the stabilization of the triple helix. In addition proline is very abundant and also exhibits a stabilizing effect on the polyproline type II helices. The denaturation temperature Tm of collagens is near to the living temperature of the organisms ${ }^{5}$ and for many collagens a monotonous relation between $\mathrm{Tm}$ and the sum of proline and hydroxyproline is observed ${ }^{6}$.

Many collagens of lower organisms are homologous to mammalian collagens? Examples are the fibril forming collagen I from annelids ${ }^{8}$ and the basement membrane

* For correspondence 
collagen IV from Drosophila ${ }^{9}$ and $C$. elegans ${ }^{10}$. On the other hand several collagens were found in invertebrates for which no resemblence exists with mammalians ${ }^{7}$. The byssus collagen of mussel ${ }^{11}$, the cuticle collagens of worms ${ }^{8}$ and the mini-collagen of nematocytes in hydra ${ }^{12}$ may serve as examples. Very interestingly, new ways of triple helix stabilization have been found for invertebrate collagens. In an annelid cuticle collagen, glycosylated threonines in Y-position replace the normally occuring hydroxyprolines ${ }^{13}$. By studies with model peptides it was found that glycosylated threonines stabilize the triple helical structure ${ }^{14}$.

Very recently, the presence of collagens in several lower organisms including phages and bacteria was proposed on the basis of cDNA sequences or genomic analysis ${ }^{15-17}$. In these cases usually no information exists about post-translational modifications like hydroxylation or glycosylation. Evidence is missing whether triple helices are formed from the Gly-Xaa-Yaa repeat sequences or not. In the present work a number of these sequences are compared and their unusual amino acid compositions are discussed from the point of view of triple helix stabilization.

\section{Results and discussion}

In table 1 several recently discovered collagen-like sequences of bacteriophages are summarized together with similar sequences of other organisms. All sequences were collected from the protein data base TrEMBL (transl.EMBL), release $5.0(2 / 98)$ and accession numbers are included to facilitate a search for further sequence information. The sequences of the phage proteins originate from genome sequencing and are frequently obtained by a combination of the sequences of several open reading frames. Sequence information on the protein level does not exist and it is therefore not known whether or not prolines are converted to hydroxyprolines or whether these or other residues are glycosylated or otherwise post-tranlationally modified. It may however be argued that phages and bacteria do not process hydroxylation and suitable glycosylation systems in their genome and that such modifications are therefore unlikely. Genetic and biochemical information is still rather incomplete but it was suggested that the collagenlike proteins are localized in the phage tail fibres ${ }^{16,17}$. An exception is a protein from bacteriophage PRD1 which was localized in the phage head ${ }^{15}$. It has a repeat of six GXY residues, which was considered a nucleation site for trimerization of this oligomeric protein.

It is not known from biochemical or biophysical data whether the putative tail fibre proteins can form homo- or hetero-trimers by triple helix formation of their collagen-like regions and what the denaturation temperatures $\mathrm{Tm}$ are. a prediction of triple helix stability by comparison with mammalian collagens cannot be made becuse of the unusual composition of residues in $\mathrm{X}$ and $\mathrm{Y}$ positions. Mammalian collagens typically contain more than $10 \mathrm{~mol} \%$ proline and an even higher fraction of hydroxyproline, together more than $20 \mathrm{~mol} \%$. In the collagenous translated sequences of the tail fibre phage BK-5T protein as an example only $6.7 \mathrm{~mol} \%$ proline are found. A striking feature of the GlyXaa-Yaa repeats in the phage proteins is the unusually high fraction of aspartate (D) and asparagine $(\mathrm{N})$ which together constitute $48 \%$ of all residues in $\mathrm{X}$ and $\mathrm{Y}$ positions in phage BK-5T (table 1). Interstingly a data base scan with $\mathrm{D}$ - and $\mathrm{N}$-rich sequence motifs revealed a number of similar sequences in bacteria, mollusks, fungi and slime mold of which examples are shown in table 1 . GNN and GDN repeats are very frequent and the total fraction of $\mathrm{D}$ and $\mathrm{N}$ residues in $\mathrm{X}$ - and $\mathrm{Y}$-position amounts to $83 \%$ in nacrein from 
Table 1 Collagen-like sequence regions in phages, viruses, bacteria, mollusks, fungi and slime mold.

\begin{tabular}{|c|c|}
\hline \multicolumn{2}{|c|}{ q38319 lactococcal phage BK-5T putative tail fiber protein } \\
\hline 0848 & GNDGKDGATGKDGVAGKDGVG \\
\hline 0940 & GNNGNDGIAGKDGVG \\
\hline 1003 & GVKGDKGDPGNNGTNGIAGKD \\
\hline 1084 & GTNGNNGHDGFPGKDGTG \\
\hline 1159 & GVKGDKGDPGNNGTNGIAGKDGKDGKG \\
\hline 1240 & GTNGNNGHDGFPGKDGTG \\
\hline 1315 & GVKGDKGDPGNNGTNGIAGKDGKG \\
\hline 1396 & GTNGNNGHDGFPGKDGTG \\
\hline 1471 & GVKGDKGDPGNNGTNGIAGKDGKG \\
\hline 1552 & GTNGNNGHDGFPGKDGTG \\
\hline 1624 & GKMGNTGPAGSNGNPGKV \\
\hline \multicolumn{2}{|c|}{ o34076 bacteriophage $\phi 01205$ putative tail fiber protein } \\
\hline 0190 & GAAGPKGDQGNDGLPGKDGVG \\
\hline 0270 & GNNGNDGLPGKDGVG \\
\hline 0342 & GEQGPKGDRGRQGLQGPRGEQGIPGPKGADGRT \\
\hline 0424 & GSDGKDGVPGKAGADGRT \\
\hline \multicolumn{2}{|c|}{ z50114 coliphage BF23 putative tail fiber protein } \\
\hline 0123 & GVDGRPGADGKPGADGKPGADGRPGDNGQRGPG \\
\hline \multicolumn{2}{|c|}{ q69475 herpesvirus putative nuclear antigen } \\
\hline 0183 & GDDGDDGDEGGDGDEGEEGQE \\
\hline \multicolumn{2}{|c|}{ q98182 molluscum contagiosum virus hypothetical protein } \\
\hline 0184 & GDDGGDGGNGGNGGDGGDGGD \\
\hline \multicolumn{2}{|c|}{006810 mycobacterium $61 \mathrm{kD}$ protein } \\
\hline 0501 & GADGTDGGKGGNGGAGGG \\
\hline 0819 & GGDGGDGGNGGN \\
\hline 0845 & GNGGDGGNGGNGGSAGTGGNGGRGGDG \\
\hline 0875 & GRNGPNGNPGGNGGAGGAGGAGLNGGNGGGAGGNGGLGGFGGN \\
\hline 1030 & GGNGGHGGHGAA \\
\hline 1043 & GGNGGPGGHGGNGGNGGTGANGGNGGIGGTGGAGSTGAKGVLGTN \\
\hline \multicolumn{2}{|c|}{ q52544 pseudomonas POPA 1 protein } \\
\hline 0186 & GVGGAGGADGGSGAGGAGGANGADGGNGVNGNQ \\
\hline \multicolumn{2}{|c|}{ q27908 oyster pearl nacrein } \\
\hline 0242 & GDNGNNGYNGDNGNNGDNGNN \\
\hline 0266 & $\begin{array}{l}\text { GDNGNNGVNGNNGYNGDNGNNGDNGNNGYNGDNGNNGDNGNNGEN } \\
\text { GNNGENGNNGENGHK }\end{array}$ \\
\hline \multicolumn{2}{|c|}{ q09164 tolypocladium inflatum cyclosporin synthase } \\
\hline \multirow{2}{*}{\multicolumn{2}{|c|}{$\begin{array}{l}15179 \text { GTNGTNGTNGTNGANGTNGTNGTNGTH } \\
\text { p90535 dyctyostelium discoidium RSC12 fragment }\end{array}$}} \\
\hline & coidium RSC12 fragment \\
\hline 0390 & GNNGNNGNNGNNGNNGNNGNNGNNGNN \\
\hline 0425 & GNNGNNGNNGNNGNNGNNGNNGNNGNNGNNGNNGQS \\
\hline
\end{tabular}

oyster pearls and almost $100 \%$ in the dyctyostelium discoidium RSC12 fragment (table 1). Aspartate and asparagine as well as threonine which also occurs in the $\mathrm{N}$ and D-rich sequences are frequently involved in complex formation with $\mathrm{Ca}^{2+}$ or other bivalent ions $^{18}$. It may therefore be hypothesized that the lack of proline and hydroxyproline is balanced by a stabilization resulting from metal ion complexation to $\mathrm{N}$ and $\mathrm{D}$ rich triple helices. Model building shows ligandation of the ions by side chains of Asp and Asn might be possible in a triple helical conformation.

Data on calcium binding so far only exist for nacrein, which is a carbonic anhydrase from the nacreous layer in oyster pearls. For this protein it was demonstrated by a 
里

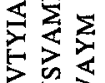

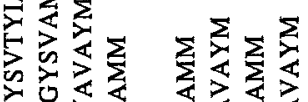

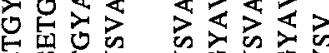

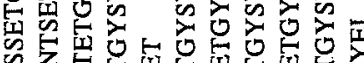

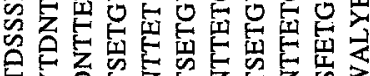

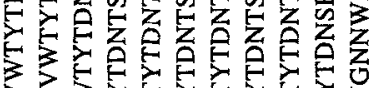

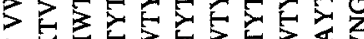

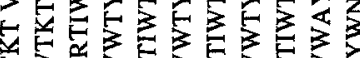

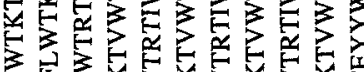

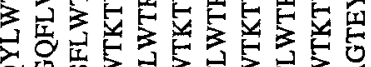

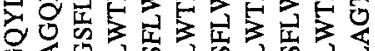

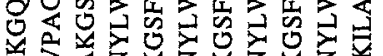

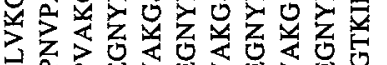

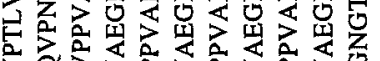

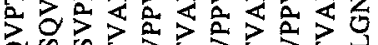

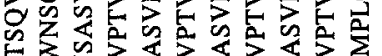

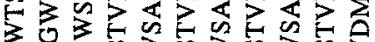

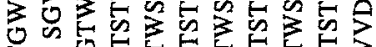

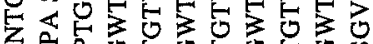

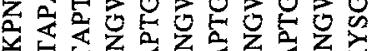

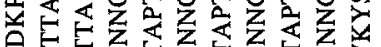

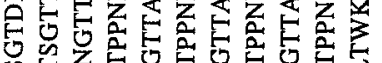

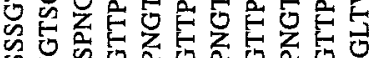

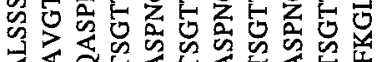

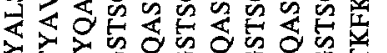

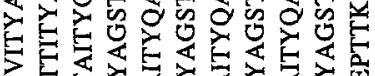

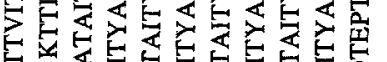

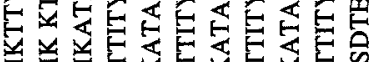

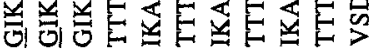

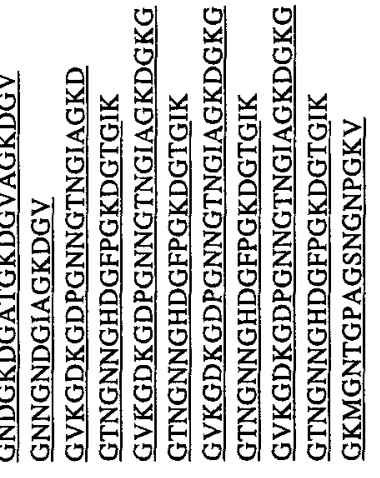

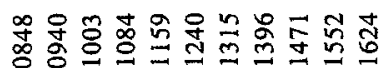

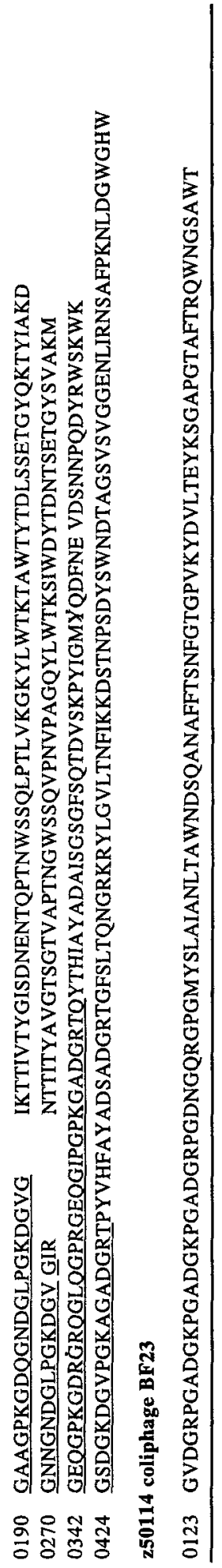


qualitative assay that $\mathrm{Ca}^{2+}$ binds to the region containing Gly-Xaa-Asn repeats with Xaa frequently being apartate ${ }^{19}$. However, our preliminary circular dichroism spectra of a synthetic peptide comprising of residues 266 to 326 in nacrein did not indicate triple helix formation in the presence or absence of calcium. A low potential for forming a triple helix was also found for synthetic pepetides in which the sequence Gly-Asn-Asn is repeated ten times. Experiments with these and related peptides will be continued introducing nucleation and cross-linking sites for promotion of triple helix formation. In the systems listed in tables 1 and 2 additional triple helix stability may originate from the adjacent globular domains. Furthermore, individual triple helices might assemble to superstructure by hydrogen bonding between glycine residues in different helices. Such supermolecular assemblies have been demonstrated for model peptides containig adjacent glycine residues. Examples are the sheet-like structures of synthetic peptides with Gly-Gly-Pro repeats and the three-dimensional assembly of polyglycine ${ }^{4,20}$.

In table 1 uninterrupted collagenous sequences are shown at their full length and all the regions following after an interruption are indicated. It can be seen that interruptions are of very variable length with the exception of the phage tail fibre proteins. They exhibit a repeat structure in which each collagen-like sequence is followed by a non-collagenous region of about 50 residues. These regions according to their amino acid composition probably form small globular domains. Within a single protein for example of phage BK$5 \mathrm{~T}$ a clear internal homology is detectable for the 50 residue repeats (table 2). Homologous domains of this type were also found in tail proteins of phages Dp-1 and $\phi$ Sfi $21^{17}$. A long stretch of 11 collagen domains, each followed by a globular domain of 50 residues is predicted with the assumption that each collagen-like sequence region forms a triple helix. The estimated length of this region in BK-5T is about $100 \mathrm{~nm}$ assuming a $0.3 \mathrm{~nm}$ translation for residues in the triple helix and a diameter of $2.5 \mathrm{~nm}$ per globular domain. Extended shapes are expected since tail fibres are very long (160 $\mathrm{nm}$ in T4 phage ${ }^{21}$ ). It should be mentioned that not all phages contain tail fibre proteins with a collagen-like sequence. The well studied $\mathrm{T} 4$ phages contain tail proteins with repeating cross $\beta$-structures. These proteins are also three-stranded ${ }^{22}$.

The repeat structure in the putative collagenous phage tail proteins were probably created by gene duplications. Repeats emerging from gene duplications were also discussed for mammalian collagens ${ }^{23,24}$ but are more difficult to prove because of the low preservation of collagen sequences not considering the glycines which repeat in every third position in all collagens. In the tail protein of phage BK5T conservation between collagenous sequences is also low but identity between the globular regions of the eleven repeats is higher than $50 \%$. This high identity suggests that the repeats are relatively recent and argues against the hypothesis that these collagens of phages are the ancestors of mammalian collagens ${ }^{16}$. For phages and bacteria, horizontal gene transfer is a likely mode of adopting a gene ${ }^{24}$. This may also be the reason why related collagen-like sequence regions are found in bacteria which are the hosts of the bacteriophages. Studies of collagens in bacteriophages are still at a very early stage. Further work will be important for a better understanding of the evolution of collagens but may also provide new insights in the stabilization of collagen triple helices.

\section{Acknowledgement}

This work was supported by a grant to JE by the Swiss National Science Foundation. 


\section{References}

1. Bateman J F, Lamandé S R and Ramshaw J A M 1996 In Collagen family in extracellular matrix (ed.) W D Comper (Amsterdam: Harwood) vol. 2 pp. 22-67

2. van der Rest M and Garrone R 1991 FASEB J. 52814

3. Hoppe H J and Reid K B 1994 Protein Sci. 8143

4. Traub W and Piez K A 1971 Adv. Protein Chemistry 25243

5. Rigby B J 1968 Nature (London) 219166

6. Josse J and Harrington W F 1964 J. Mol. Biol. 9269

7. Engel J 1997 Science 2771785

8. Gaill F, Wiedemann H, Mann K, Kühn K, Timpl R and Engel J 1991 J. Mol. Biol. 221209

9. Blumberg B, MacKrell A J and Fessler J H 1988 J. Biol. Chem. 26318328

10. Kramer J M 1994 FASEB J. 8329

11. Coyne K J, Qin X X and Waite J H 1997 Science 2771830

12. Kurz E M, Holstein T W, Petri B M, Engel J and David C N 1991 J. Cell Biol. 1151159

13. Mann K, Mechling D E, Bächinger H P, Eckerskorn C, Gaill F and Timpl R 1996 J. Mol. Biol. 16255

14. Bann $J E$ and Bächinger $H P$ (unpublished results)

15. Bamford J K H and Bamford D H 1990 Virology 177445

16. Smith M C M, Burns N, Sayers J R, Sorrell J A, Casjens R S and Hendrix R W 1998 Science 2791834

17. Diesiere F, Lucchini S and Brüssow H 1998 Virology 241345

18. Maurer P, Hohenester E and Engel J 1996 Curr. Opinion Cell Biol. 8609

19. Miyamoto H, Miyashita T, Okushima M, Nakano S, Morita T and Matsushiro A 1996 Proc. Natl. Acad. Sci. USA 939657

20. Traub W, Yonath A and Segal D M 1969 Nature (London) 221914

21. Revel H R 1981 In Bacteriophage assembly (ed.) M S DuBow (New York: Liss) pp. 353-364

22. Earnshaw W C, Goldberg E B and Crowther R A 1979 J. Mol. Biol. 132101

23. Saitta B, Wang Y M, Renkart L, Zhang R Z, Pan T C, Timpl R and Chu M L 1991 Genomics 11145

24. Doolitle R F 1992 Protein Sci. 1191 